\title{
AN OPERATOR ERGODIC THEOREM FOR SEQUENCES OF FUNCTIONS ${ }^{1}$
}

\section{E. M. KLIMKO AND LOUIS SUCHESTON}

1. Introduction. In 1940 P. T. Maker [7] showed that in Birkhoff's ergodic theorem images of a single function $f$ can be replaced by images of functions forming a double sequence, dominated by an integrable function and converging to $f$. Here we obtain an analogous generalization of the Chacon-Ornstein ratio ergodic theorem for operators. It is also shown by example that dominated sequences in general cannot be replaced by uniformly integrable sequences.

In $1957 \mathrm{~L}$. Breiman applied Maker's theorem to establish "an ergodic theorem of information theory," (see [2] and [5]). An analogous application of a variant of our result is given in [6].

2. The theorem. Let $(X, a, \mu)$ be a $\sigma$-finite measure space. We consider semi-Markovian operators: positive linear operators mapping $L_{1}$ into $L_{1}$. If the $L_{1}$ norm of $T$ is less than or equal to one, $T$ is called sub-Markovian. The aspects of the theory of sub-Markovian operators of interest for us are developed e.g. in [8]; the assumption made there that $\mu(X)=1$ is, for most purposes, inessential.

All relations below are to be understood modulo sets of measure zero. By $L_{1}^{+}$we denote the class of nonnegative, not identically vanishing functions of $L_{1}$. The operator $I+T+T^{2}+\cdots$ is written $T_{\infty}$. We let $A$ be a measurable subset of $X$, such that on $A$ the operator $T$ is conservative, the ratio theorem holds and the limit is well behaved. More precisely, we assume the following conditions:

$\left(c_{A}\right) T_{\infty} g=\infty$ or 0 on $A$ for each $g \in L_{1}^{+}$and

$\left(r_{A}\right)$ If $f \in L_{1}^{+}, g \in L_{1}^{+}$, then on the set $A \cap\left\{T_{\infty} g>0\right\}$

$$
D(f, g) \stackrel{\text { def }}{=} \lim _{n \rightarrow \infty} \sum_{i=0}^{n-1} T^{i f} / \sum_{i=0}^{n-1} T^{i} g
$$

exists and is finite. Further, if $F_{k} \in L_{1}, F_{k} \downarrow 0(k \rightarrow \infty)$ on $X$, then for each $g \in L_{1}^{+}, D\left(F_{k}, g\right) \rightarrow 0$ on the set $A \cap\left\{T_{\infty} g>0\right\}$.

The conditions $\left(c_{A}\right)$ and $\left(r_{A}\right)$ are satisfied if $T$ is a sub-Markovian operator and $A$ is the conservative part $C$ of the space. We only verify $\left(r_{A}\right)$. If $\mu(X)=1$, then on the set $C \cap\left\{T_{\infty} g>0\right\}$ one has

$$
D\left(F_{k}, g\right)=E\left(R F_{k} / \mathfrak{e}\right) / E(R g / \mathfrak{e})
$$

Received by the editors August 1, 1967.

${ }_{1}^{1}$ Research supported by National Science Foundation Grant GP 7693. 
where $\mathcal{C}$ is the $\sigma$-field of invariant sets and the operator $R$ adds to the function $f \cdot 1_{c}$ the total contribution of the dissipative part $D$, (see [4], [3], [8, p. 211]). Since $R$ and $E(\cdot / \mathfrak{e})$ are sub-Markovian operators, the last assertion in $\left(r_{A}\right)$ follows from the monotone continuity theorem for such operators (see [8, p. 187]). If $\mu$ is a $\sigma$-finite measure, we let $\pi$ be an equivalent probability measure and $p=d \pi / d \mu$, the Radon-Nikodým derivative of $\pi$ with respect to $\mu$. Now define an operator $U$ by $U g=(1 / p) \cdot T(p \cdot g)$ where $g \in L_{1}(\pi)$, or, equivalently, $p \cdot g \in L_{1}(\mu)$. The passage to $\pi$ and $U$ leaves the ratios in $\left(r_{A}\right)$ invariant; hence the $\sigma$-finite case reduces to the case $\mu(X)=1$. More generally, if $T$ is a semi-Markovian operator satisfying the boundedness assumption $\left(b_{h}\right)$, then the conditions $\left(c_{A}\right)$ and $\left(r_{A}\right)$ hold if $A$ is the conservative part $Y C^{h}$ of the set $Y^{h}$ (see [9]).

We now state our theorem.

THEOREM 1. Assume that $T$ is a semi-Markovian operator satisfying for some set $A$ the conditions $\left(c_{A}\right)$ and $\left(r_{A}\right)$. Let $f_{n i}$ and $g_{n i}, n, i=0$, $1, \cdots$, be double sequences of functions in $L_{1}^{+}$such that $\lim _{n, i} f_{n i}$ $=f, \lim _{n, i} g_{n i}=g$ and

$$
\sup _{n, i} f_{n i} \in L_{1}^{+}, \quad \sup _{n, i} g_{n i} \in L_{1}^{+} .
$$

Then on the set $A \cap\left\{T_{\infty} g>0\right\}$ one has

$$
\lim _{n \rightarrow \infty} \frac{\sum_{i=0}^{n-1} T^{i} f_{n i}}{\sum_{i=0}^{n-1} T^{i} g_{n i}}=D(f, g) .
$$

Proof. It suffices to show that

$$
\limsup _{n \rightarrow \infty}\left|\frac{\sum_{i=0}^{n-1} T^{i} f_{n i}}{\sum_{i=0}^{n-1} T^{i} g_{n i}}-\frac{\sum_{i=0}^{n-1} T^{i f}}{\sum_{i=0}^{n-1} T^{i} g}\right|=0 .
$$

We have

$$
\left|\frac{\sum_{i=0}^{n-1} T^{i} f_{n i}}{\sum_{i=0}^{n-1} T^{i} g}-\frac{\sum_{i=0}^{n-1} T^{i} f}{\sum_{i=0}^{n-1} T^{i} g}\right| \leqq \frac{\sum_{i=0}^{n-1} T^{i}\left|f_{n i}-f\right|}{\sum_{i=0}^{n-1} T^{i} g} .
$$


For each fixed $M, N$ let

$$
F_{N M}=\sup _{n \geqq N, m \geqq M}\left|f_{n m}-f\right| .
$$

Then for $n>N$

$$
\frac{\sum_{i=0}^{n-1} T^{i}\left|f_{n i}-f\right|}{\sum_{i=0}^{n-1} T^{i} g} \leqq \frac{\sum_{i=0}^{M-1} T^{i} F_{00}}{\sum_{i=0}^{n-1} T^{i} g}+\frac{\sum_{i=M}^{n-1} T^{i} F_{N M}}{\sum_{i=0}^{n-1} T^{i} g} .
$$

Therefore

$$
\begin{gathered}
\limsup _{n \rightarrow \infty}\left|\frac{\sum_{i=0}^{n-1} T^{i} f_{n i}}{\sum_{i=0}^{n-1} T^{i} g}-\frac{\sum_{i=0}^{n-1} T^{i f}}{\sum_{i=0}^{n-1} T^{i} g}\right| \\
\leqq \underset{n \rightarrow \infty}{\lim \sup } \frac{\sum_{i=0}^{M-1} T^{i} F_{00}}{\sum_{i=0}^{n-1} T^{i} g}+\underset{n \rightarrow \infty}{\lim \sup } \frac{\sum_{i=M}^{n-1} T^{i} F_{N M}}{\sum_{i=0}^{n-1} T^{i} g} .
\end{gathered}
$$

By $\left(c_{A}\right)$ the first term in (7) is zero on the set $A \cap\left\{T_{\infty} g>0\right\}$ while by $\left(r_{A}\right)$ the second term is $D\left(F_{N M}, g\right)$ on the same set. Since $F_{N M} \leqq F_{k k}$ $\downarrow 0(N, M \rightarrow \infty)$ where $k=\min (N, M)$, again by $\left(r_{A}\right)$ we have that $D\left(F_{N M}, g\right) \rightarrow 0$ on $A \cap\left\{T_{\infty} g>0\right\}$; hence the expression (6) is zero, which proves the particular case of (3) when all the functions $g_{n i}$ equal $g$. (3) follows because

$$
\frac{\sum_{i=0}^{n-1} T^{i} f_{n i}}{\sum_{i=0}^{n-1} T^{i} g_{n i}}=\frac{\sum_{i=0}^{n-1} T^{i} f_{n i}}{\sum_{i=0}^{n-1} T^{i} g} \cdot \frac{\sum_{i=0}^{n-1} T^{i} g}{\sum_{i=0}^{n-1} T^{i} g_{n i}}
$$

and the last ratio converges to $1 / D(g, g)=1$. This completes the proof of the theorem.

REMARK. The proof shows that if the assumption $\left(r_{A}\right)$ is weakened by replacing the limit $D$ by the limit superior, one still has the following conclusion: on $A \cap\left\{T_{\infty} g>0\right\}$ 


$$
\underset{n}{\lim \sup } \frac{\sum_{i=0}^{n-1} T^{i} f_{n i}}{\sum_{i=0}^{n-1} T^{i} g}=\lim \sup _{n} \frac{\sum_{i=0}^{n-1} T^{i} f}{\sum_{i=0}^{n-1} T^{i} g},
$$

and an analogous equality holds for the limit inferior.

The following corollary, concerned with single sequences, may be considered as a generalization of Hopf's decomposition theorem (see $[8$, p. 196]). This theorem asserts that the space $X$ decomposes into the conservative part $C$ and the dissipative part $D$ : for each $g \in L_{1}^{+}$, $T_{\infty} g=\infty$ on $C \cap\{g>0\}, T_{\infty} g<\infty$ on $D$.

Corollary 1. Let $T$ be a sub-Markovian operator, let $g_{i} \rightarrow g$ as $i \rightarrow \infty, g_{i} \in L_{1}^{+}, \sup _{i} g_{i} \in L_{1}^{+}$. Then

$$
\lim _{n \rightarrow \infty} \sum_{i=0}^{n-1} T^{i} g_{i}
$$

is infinite on the set $C \cap\{g>0\}$ and is finite on the set $D$.

Proof. The assertion about the behavior on $C$ follows from Theorem 1 applied with $A=C, f_{n i}=f>0, f \in L_{1}, g_{n i}=g_{i}$. On the other hand, on $D$ we have

$$
\lim _{n \rightarrow \infty} \sum_{i=0}^{n-1} T^{i} g_{i} \leqq \lim _{n \rightarrow \infty} \sum_{i=0}^{n-1} T^{i}\left(\sup _{j} g_{j}\right)
$$

which is finite by Hopf's theorem.

For semi-Markovian operators the corollary remains valid with $C$ replaced by $Y C^{h}, D$ replaced by $Y D^{h}$.

3. A counterexample. Is it possible to replace the assumption (1) in Theorem 1 by the weaker assumption of uniform integrability of sequences? We particularize as follows. Let $\left(v_{i}\right),\left(p_{i}\right), i=1,2, \cdots$, be sequences of positive constants satisfying

$$
\sum_{i} p_{i}=1, \quad v_{n} p_{n} \rightarrow 0, \quad \sum_{n} v_{n} p_{n} / n=\infty .
$$

Now let $(X, a, \mu)$ be a probability space such that there is a measurable partition $\left\{A_{i}\right\}$ of $X$ with $\mu\left(A_{i}\right)=p_{i}, i=1,2, \cdots$. Let $g_{n i}=1$ for all $n, i$ and let

$$
\begin{aligned}
f_{n i}=f_{i} & =v_{i} & & \text { on } A_{i} \\
& =0 & & \text { on } X-A_{i} .
\end{aligned}
$$


The sequence $f_{i}$ converges to $f=0$. The uniform integrability in the presence of pointwise convergence to zero is equivalent with the convergence of the integrals $\int f_{i} d \mu=v_{i} p_{i}$ to $\int 0 d \mu=0$; thus $\left(f_{i}\right)$ is uniformly integrable. Moreover, on $A_{n}$

$$
\sup _{n} \sum_{i=1}^{n} \frac{f_{i}}{n}=\sup _{n} \frac{f_{n}}{n}=\frac{v_{n}}{n}
$$

hence

$$
\int\left(\sup _{n} \sum_{i=1}^{n} f_{i} / n\right) d \mu=\sum_{n} v_{n} p_{n} / n=\infty .
$$

By a theorem of Blackwell and Dubins [1], there is, on a suitable probability space $\left(X^{*}, a^{*}, \mu^{*}\right)$, a sequence of functions $\left(f_{i}^{*}\right)$ with the same joint distribution as $\left(f_{i}\right)$, and a $\sigma$-field $\mathcal{e}$ such that

$$
\mu^{*}\left\{\frac{1}{n} \sum_{1}^{n} E\left(f_{i} \mid \mathcal{e}\right) \rightarrow 0\right\}=0 .
$$

We identify the starred and the nonstarred expressions. Let the operator $T$ be the conditional expectation $E(\cdot \mid \mathfrak{e})$. Then $T 1=1$, $T^{2}=T, T$ satisfies the assumptions $\left(c_{A}\right)$ and $\left(r_{A}\right)$ with $A=X$, but by (15) the equality (2) fails on the entire space $X$.

\section{REFERENCES}

1. D. Blackwell and L. E. Dubins, $A$ converse to the dominated convergence theorem, Illinois J. Math. 7 (1963), 508-514.

2. L. Breiman, The individual ergodic theorem of information theory, Ann. Math. Statist. 28 (1957), 809-811. Correction note, ibid. 31 (1960), 809-810.

3. R. V. Chacon, Identification of the limit of operator averages, J. Math. Mech. 11 (1962), 957-961.

4. R. V. Chacon and D. S. Ornstein, A general ergodic theorem, Illinois J. Math. 4 (1960), 153-160.

5. A. Ionescu Tulcea, Contributions to information theory for abstract alphabets, Ark. Mat. 4 (1961), 235-247.

6. E. M. Klimko and L. Sucheston, On convergence of information in spaces with infinite invariant measure, Z. Wahrscheinlich keitstheorie und Verw. Gebiete 10 (1968), 226-235.

7. P. T. Maker, The ergodic theorem for a sequence of functions, Duke Math. J. 6 (1940), 27-30.

8. J. Neveu, Mathematical foundations of the calculus of probability, Holden-Day, San Francisco, Calif., 1965.

9. L. Sucheston, On the ergodic theorem for positive operators. I, Z. Wahrscheinlichkeitstheorie Verw. Gebiete 8 (1967), 1-11; II, ibid. 8 (1967), 353-356.

Ohio State University 